\title{
Crystallographic Structure Determination of MFI-Zeolite Nanosheets.
}

\author{
Prashant Kumar, ${ }^{1}$ Kumar Varoon Agrawal, ${ }^{1}$ Michael Tsapatsis, ${ }^{1}$ K. Andre Mkhoyan ${ }^{1}$ \\ ${ }^{1}$ Department of Chemical Engineering \& Materials Science, University of Minnesota, Minneapolis, MN
}

Ultrathin MFI-zeolite nanosheets can be packed on porous supports to create molecular sieve membranes. The nanosheets on porous support reduce morphological damage seen in regular MFIzeolite crystals [1]. MFI-zeolite belongs to pentasil family of zeolites where the periodic building unit (PerBU) is composed of 12 interconnected $\mathrm{SiO}_{4}$ tetrahedral (T12) units of $4_{1} \mathrm{~m} 2\left(\mathrm{D}_{2 \mathrm{~d}}\right)$ symmetry [2]. Rotation of T12-units about c-axis, along with translation by 0.5 unit cell in [001] direction forms righthanded or left-handed pentasil chains consisting of five membered rings. Alternating left- and righthanded chains, when connected along [100] direction through inversion symmetry, form MFI-zeolite structure.

To resolve the crystal structure of MFI-zeolite nanosheets, we performed bright-field conventional transmission electron microscopy (BF-CTEM) and high angle annular dark-field scanning transmission electron microscopy (HAADF-STEM) imaging using FEI Tecnai $\mathrm{G}^{2}$ F30 (S)TEM operated at optimum $200 \mathrm{kV}$ [3]. Data was collected using low dose of electrons to avoid beam damage of these nanosheets [4], thus resulting in low signal to noise ratio (SNR) in the images. In order to improve structural visibility in TEM image, BF-CTEM image was digitally processed using wiener filter to reduce the noise and frame-averaged using cross-correlation algorithm to resolve the pentasil chains of MFI-zeolite nanosheets (Figure 1c).

In order to measure the thickness of the MFI-zeolite nanosheets along [010] direction (perpendicular to sheet) we collected selected area diffraction patterns from 0 to $20^{\circ}$ tilts with $2^{\circ}$ steps about [301] axis. Simulations of the same diffraction patterns using multislice method [5] with TEMSIM code [6] for MFI-zeolite nanosheets with 0.5, 1.0, 1.5 and 2.0 unit cell thicknesses revealed that our nanosheets are 1.5 unit cell thick (Figure 2). HAADF-STEM images further revealed that almost all synthesized MFIzeolite nanosheets have same thickness; two nanosheets stacked on top of each other are in coherence with doubling of Z-contrast in the HAADF-STEM image intensity (Figure 1b) [7].

\section{References:}

[1] K. Varoon et al, Science 334 (2011), 72-75.

[2] G.T. Kokotailo et al, Nature 275 (1978), 119-120.

[3] K. Yoshida et al, Microscopy 62 (2013), 369-375.

[4] O. Ugurlu et al, Phys. Rev. B 83 (2011), 113408.

[5] J.M. Cowley, A.F. Moodie, Acta Cryst. 10 (1957), 609-619.

[6] E.J. Kirkland in "Advanced Computing in Electron Microscopy", ed. 2, (Springer, New York).

[7] The authors acknowledge funding by the MRSEC Program of the National Science Foundation under Award Number DMR-0819885. 

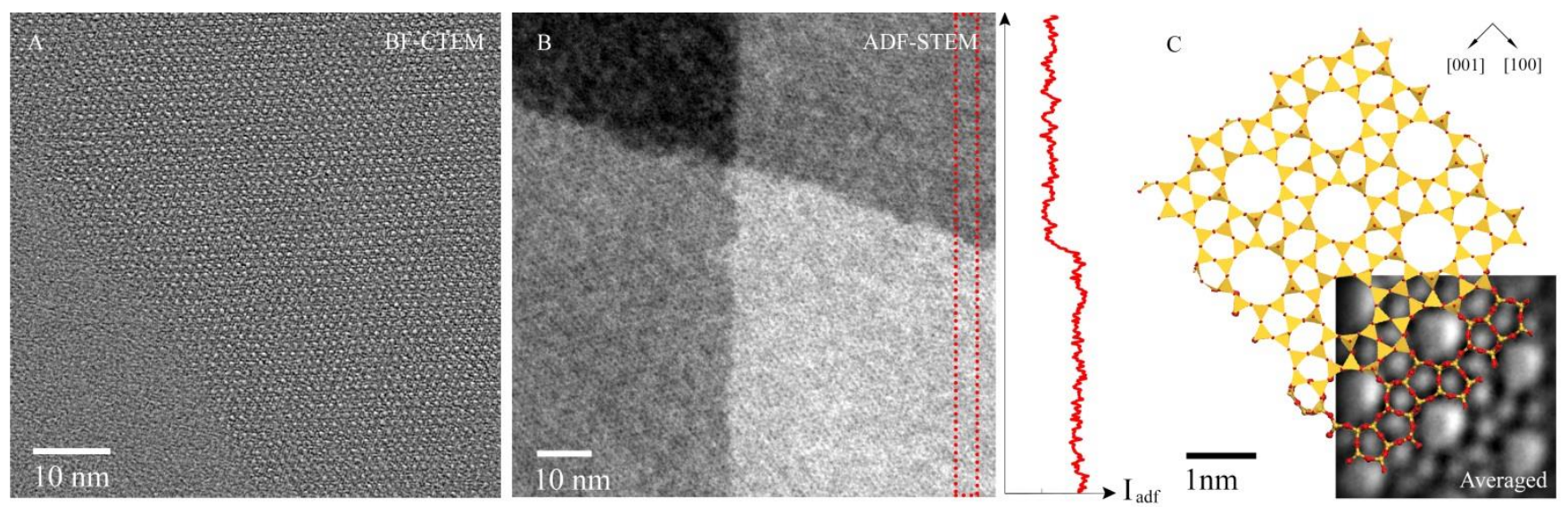

Figure 1. (a) Wiener filtered BF-CTEM image of b-projection of MFI nanosheet, (b) HAADF-STEM image showing two nanosheets stacked on top of each other with the rectangular box showing an intensity line scan across the image, (c) frame averaged section of the original BF-CTEM image overlaid with MFI crystal structure.

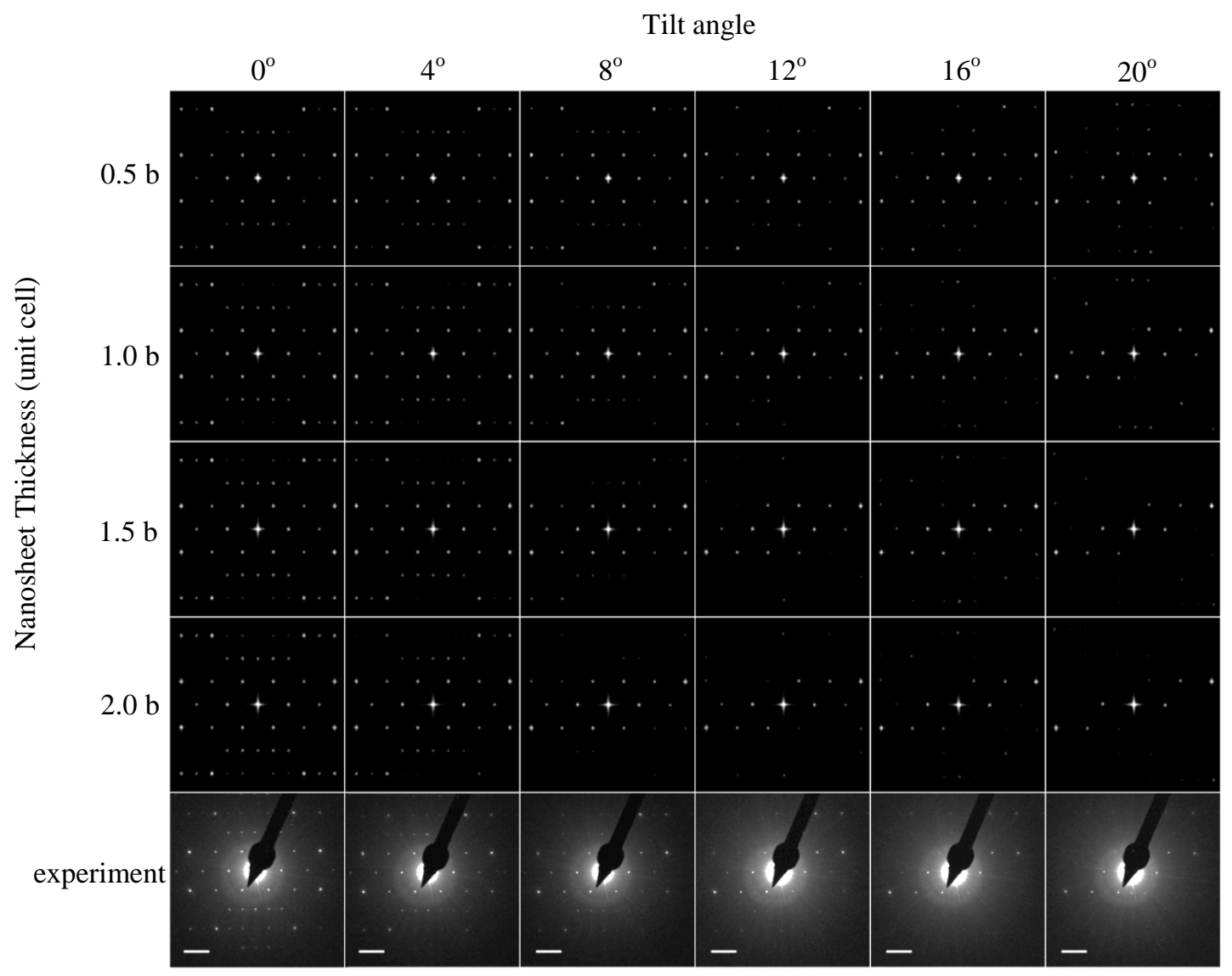

Figure 2. A tilt series of diffraction patterns simulated using multislice code for thickness of $0.5,1.0$, 1.5 and 2.0 unit cell in [010] direction when compared with experimentally obtained SAD patterns shows a close match for a thickness of 1.5 unit cell in [010] direction (scale $1 \mathrm{~nm}^{-1}$ ). 